\title{
Looking into Lyme in the mouse brain
}

Casselli, T. et al. PLoS Pathog 17, e1009256 (2021)

While Timothy Casselli was completing his $\mathrm{PhD}$ studying Lyme disease at Washington State University, he encountered a common refrain: mice don't get central nervous system (CNS) involvement. Since the 1990s, there have been mouse models of Lyme disease in which to study the joint and heart inflammation that can result from infections with tick-borne Borrelia spirochetes, but studying the neurological manifestations - the pain, headache, and fatigue that, in some Lyme patients, can linger long after they've finished the course of antibiotics to eliminate active infections remained out of reach in mice.

But after decades of assumptions that the bacteria just couldn't make it into the CNS, it turns out the mouse brain isn't just a naïve bystander during Lyme infections after all. "People just weren't looking in precisely the right place," says Casselli's colleague, Catherine Brissette, a fellow Lyme researcher at the University of North Dakota.

A couple years ago, Ali Divan, a post-doc working with Casselli and Brissette who came from an immunology lab, decided to try out some different combinations of parameters to mimic tick infection. He serendipitously chose to inject the mice - the same $\mathrm{C} 3 \mathrm{H}$ strain long used to model Lyme-associated arthritis and carditis - with different strains of Borrelia burgdorferi intradermally near the shoulder blade, rather than the base of the tail. Nor did he limit sampling for the pathogenic spirochetes to the brain itself. Using a method to separate out the meningeal tissue that surrounds the brain and spinal cord and traffics leukocytes between the CNS and lymph nodes, he found spirochetes for up to 75 days following infections squirming about in the animals' dura mater - the outmost layer of the meninges. New work from the group recently published in
PLoS Pathogens further characterizes the kinetics of Lyme disease infections and the associated immune responses in both the mouse meninges and brain.

The team found that Borrelia burgdorferi concentrations peaked in the dura mater after a week following infection, and that that indeed varied based on inoculation site - the closer to the brain, the higher the numbers. They also observed localized inflammatory responses in the dura mater of infected animals that were similar to prior observations in the joints and hearts of mice modeling Lyme arthritis and carditis. These responses include upregulation of genes involved in cytokine expression and the interferon response, leukocyte infiltration, and vascular changes in infected animals. The brain, however, remained free of both spirochetes and leukocytes, and the only notable upregulation of immune system genes was in those involved in the interferon response.

The response in the brain may have been subtle, but that's not entirely unexpected given how Lyme disease manifests in people. "In arthritis, you see a lot of immune cells showing up, you see a lot of destruction of cartilage, sometimes you even see some bone loss," says Casselli. "If you have the kind of inflammation in the brain as you are seeing in the joints, this would not just be problems with fatigue and headache you'd be in serious trouble." Rather, patients suffering from Lyme neuroborreliosis - the term for those neurologic symptoms that can follow Lyme disease - share similar complaints to others being treated with interferon for various unrelated conditions; both describe experiencing memory trouble, fatigue, and 'brain fog.'

"The brain is showing a definite immune response, but there's no bacteria there," says Casselli. "That could definitely inform how we're interpreting what neuroborreliosis is."

Symptoms such as fatigue and brain fog are difficult to quantify in people, let alone in mice - not to mention that many of the assays to measure what comparable phenotypes there are in the animals require them to move, which in Lyme models can be complicated by the concurrent arthritis the disease can produce. Studying more behavioral and clinical manifestations of Lyme disease will remain in the realm of rhesus macaques, Casselli and Brissette say, for which there is a model that more closely recapitulates human Lyme disease symptoms.

Rather, the ability to now study CNS involvement in Lyme disease in mice should help Lyme researchers answer mechanistic questions and better understand the factors that lead to neurological involvement when it does turn up in Lyme disease patients - details such as what Borrelia strains or species are most likely to lead to neuroborreliosis, whether there are environmental influences that add to the burden, and what host factors might contribute to disease outcomes. If a patient presents with a particular Borrelia strain shown to invoke stronger CNS involvement in mouse than other varieties, that may warrant more aggressive treatment, for example.

"The mouse model is going to be really, really useful in developing a lot of testable hypotheses," says Brissette, the answers to which can then help inform nonhuman primate studies as well as human trials.

\section{Ellen Neff}

Published online: 17 March 2021

https://doi.org/10.1038/s41684-021-00744-9 